\title{
Human Osteoblasts In Vitro Secrete Tissue Inhibitor of Metalloproteinases and Gelatinase but Not Interstitial Collagenase as Major Cellular Products
}

\author{
L. Rifas, L. R. Halstead, W. A. Peck, L. V. Avioli, and H. G. Welgus* \\ Division of Bone and Mineral Metabolism and *Division of Dermatology, Department of Medicine, \\ Jewish Hospital at Washington University Medical Center, St. Louis, Missouri 63110
}

\begin{abstract}
Human osteoblast cultures (hOB) were examined for the production of interstitial collagenase, tissue inhibitor of metalloproteinases (TIMP), and gelatinolytic enzymes. Cells were isolated by bacterial collagenase digestion of trabecular bone (vertebra, rib, tibia, and femur) from 11 subjects (neonatal to adult). Confluent cultures were exposed to phorbol 12-myristate 13-acetate, PTH, PGE 2 , epidermal growth factor, $1,25(O H)_{2}$ vitamin $D_{3}$, recombinant human IL-1 $\beta$, and dexamethasone. Collagenase and TIMP were assayed immunologically and also by measurements of functional activity. Collagenase was not secreted in significant quantities by human bone cells under any tested condition. Furthermore, collagenase mRNA could not be detected in hOB. However, hOB spontaneously secreted large amounts of TIMP for at least $72 \mathrm{~h}$ in culture. hOB TIMP was found to be identical to human fibroblast TIMP by double immunodiffusion, metabolic labeling and immunoprecipitation, Northern blot analysis, and stoichiometry of collagenase inhibition. SDS-substrate gel electrophoresis of hOB-conditioned media revealed a prominent band of gelatinolytic activity at $68 \mathrm{kD}$, and specific polyclonal antisera established its identity with the major gelatinolytic protease of human fibroblasts. Abundant secretion of gelatinolytic, but not collagenolytic, enzymes by hOB may indicate that human osteoblasts do not initiate and direct the cleavage of osteoid collagen on the bone surface, but may participate in the preparation of the bone surface for osteoclast attachment by removal of denatured collagen peptides. The constitutive secretion of TIMP may function to regulate metalloproteinase activity.
\end{abstract}

\section{Introduction}

Bone is a complex organ composed of many cell types including cells of mesenchymal origin as well as those of the immune system (1). The growth of bone begins with a superstructure formed either from cartilage (endochondral ossification, e.g., long bones) or connective tissue (intramembranous ossification, e.g., flat bones). During the course of development, osteo-

\footnotetext{
This work was presented in part in 1987 at the Ninth Annual Scientific Meetings of the American Society for Bone and Mineral Research in Indianapolis, IN.

Received for publication 2 May 1988 and in revised form 16 March 1989.
}

J. Clin. Invest.

(C) The American Society for Clinical Investigation, Inc. 0021-9738/89/08/0686/09 \$2.00

Volume 84, August 1989, 686-694 blasts arise and infiltrate the superstructure, depositing matrix proteins including type I collagen, proteoglycans, fibronectin, osteonectin, and many others $(2,3)$. This milieu is termed osteoid. After the osteoid is deposited by the osteoblasts, it becomes calcified, yielding the hard connective tissue of bone. During this process, some osteoblasts become embedded within the bone matrix and are called osteocytes, cells that help to maintain the integrity of bone. New bone is modeled by the activity of osteoclasts, cells of bone marrow mononuclear cell origin that fuse into multinucleated cells (4-6). These specialized cells are capable of removing calcified osteoid and are thus responsible for the formation of the bone marrow cavity. Within the marrow cavity, the osteoblasts form islands of bone called trabeculae which give strength to the bone. Once formed, mature bone undergoes virtually continuous remodeling throughout the life of the organism. Teams of osteoclasts resorb microscopic quanta of tissue from the bony surfaces and, subsequently, osteoblasts are recruited to repair the resorption defect (7).

When bone formation is complete, the osteoblasts then become quiescent and flatten out over the surface of bone. To initiate remodeling, it has been hypothesized that the flattened osteoblasts contract under the influence of resorption stimulative hormones such as PTH or $\mathrm{PGE}_{2}$, creating a void in the lining of the bone where osteoclasts attach and resorb the calcified matrix (8-11). In addition, in vitro studies have suggested that a thin layer of organic matrix which lines the mineralized bone surface must be removed in order for osteoclasts to be attracted and attach to the newly exposed surface (12, 13). It has been hypothesized from studies on rodent bone cultures that osteoblasts, under the influence of bone-resorption-stimulating hormones, secrete interstitial collagenase which subserves the degradation of surface type I collagen and thereby promotes osteoclast attraction and attachment (12-18). Rodent osteoblasts have also been reported to secrete a protease inhibitor which may block the action of collagenase and perhaps other metalloenzymes $(14,19)$, thereby providing more precise regulatory control over the process.

We have conducted this study to determine whether human osteoblasts in homogeneous monolayer culture are capable of secreting collagenase and tissue inhibitor of metalloproteinases (TIMP). ${ }^{1}$ Our results indicate that human osteoblasts do not secrete a neutral interstitial collagenase in significant quantities, either constitutively or under the influence of bone-resorbing hormones, but do secrete gelatinolytic pro-

1. Abbreviations used in this paper: BGP, bone Gla protein; EGF, epidermal growth factor; hOB, human osteoblast cultures; rhIL-1 $\beta$, recombinant human interleukin $1 \beta$; TIMP, tissue inhibitor of metalloproteinases. 
teases. These cells produce substantial amounts of TIMP, which may act to regulate metalloproteinase activity within the local environment.

\section{Methods}

\section{Materials}

PTH (human, fragment 1-34) and PGE $_{2}$ were obtained from Sigma Chemical Co. (St. Louis, MO). 1,25(OH) $)_{2}$ vitamin $\mathrm{D}_{3}$ was a kind gift of Dr. Milan R. Uskokovic, Hoffman-LaRoche Inc. (Nutley, NJ), and EGF was purchased from Collaborative Research (Boston, MA). Recombinant interleukin $1 \beta$ was obtained from Genzyme (Boston, MA), and phorbol-12 myristate 13-acetate (PMA) was obtained from L.C. Services Corporation (Woburn, MA). All other reagents were purchased from Sigma Chemical Co.

\section{Methods}

Cell cultures. Human bone cell cultures were prepared according to the method of Robey and Termine, with modification (2). Briefly, surgical specimens of rib were split longitudinally and the marrow cavity rinsed extensively with serum-free medium (DME/Ham's F-12, 1:1, vol:vol). The trabecular bone was removed with a size 0 curette, minced and washed several times. The bone chips were then subjected to bacterial collagenase digestion $\left(2 \mathrm{mg} / \mathrm{ml}\right.$, type IV) for $2 \mathrm{~h}$ at $27^{\circ} \mathrm{C}$. The released cells were washed three times and then seeded into T-75 flasks (Costar, Cambridge, MA) in medium supplemented with 10\% FBS (Sigma Chemical Co.). After reaching confluency, the cells were removed with trypsin-EDTA (0.05-0.02\%, respectively, Sigma Chemical Co.), counted, and seeded at $2.5 \times 10^{4}$ cells per $16-\mathrm{mm}$ well of 24-well tissue culture plates (Costar) in 10\% FBS-containing medium. The cells were then allowed to grow to confluency (4-8 wk) with medium changes every 3-4 d.

Normal human skin fibroblasts were purchased from American Type Culture Collection (Rockville, MD). The cells were grown in Dulbecco's modified Eagle's medium-HG + glutamine, 0.03 M Hepes buffer (pH 7.6), $10 \%$ fetal calf serum, and $200 \mathrm{U} / \mathrm{ml}$ of penicillin +200 $\mu \mathrm{g} / \mathrm{ml}$ of streptomycin. After two to three passages, the cells were grown to visual confluence, washed with HBSS, and cultured under serum-free conditions for $48 \mathrm{~h}$.

Bone cell-conditioned medium. Confluent cell cultures were washed with fresh medium and the following hormones or chemicals added for the time period indicated in the relevant figure legends or tables: PMA, $1 \times 10^{-8} \mathrm{M}$; PTH, human $1-34,1 \times 10^{-8}$ to $1 \times 10^{-7} \mathrm{M}$; $1,25(\mathrm{OH})_{2}$ vitamin $\mathrm{D}_{3}, 1 \times 10^{-9} \mathrm{M}$; epidermal growth factor (EGF), 10 $\mathrm{ng} / \mathrm{ml}$; recombinant human interleukin $1 \beta$ (rhIL-1 $\beta), 1-10 \mathrm{U} / \mathrm{ml}$; and $\mathrm{PGE}_{2}, 1 \times 10^{-8} \mathrm{M}$. The conditioned media were collected and assayed for collagenase and TIMP by ELISA (see below). To obtain samples for the assay of collagenase and TIMP by functional activity, cultures were washed three times and refed with serum-free medium prior to the addition of the appropriate agents.

\section{Assays}

Assays for alkaline phosphatase. Human osteoblast cultures were grown to confluency in serum-containing medium in 16-mm wells of 24-well tissue culture plates. The cultures were then washed three times with phosphate-buffered saline, $\mathrm{pH} 7.4$, and lysed in water by three freeze-thaw cycles. The lysates were then homogenized by sonication (Dismembranator model 150, Oscar Fisher, Inc., Newburgh, NY) for $10 \mathrm{~s}$. The protein concentration and alkaline phosphatase activity in each lysate were measured by the Coomassie blue protein assay (BioRad Laboratories, Richmond, CA) and an alkaline phosphatase kit (No. 104-LL, Sigma Chemical Co.) respectively. The enzymatic activity was expressed as nanomoles per minute per milligram of protein. Sister cultures were stained for alkaline phosphatase using a histochemical kit (No. 85L-2, Sigma Chemical Co.).

Assay for CAMP. Assays for cAMP were performed as previously described (2).
Radioimmunoassay for bone Gla protein (BGP). Confluent cultures were washed and incubated for $24 \mathrm{~h}$ in serum-free medium in the presence of $1 \mathrm{nM} 1,25-(\mathrm{OH})_{2}$ vitamin $D_{3}$. Supernatants were assayed for BGP using a radioimmunoassay kit obtained from Incstar (Stillwater, MA).

Immunologic assays of collagenase and TIMP. Collagenase and TIMP levels in the various conditioned media were measured by ELISA using specific polyclonal antisera prepared to the respective human fibroblast proteins as described previously $(20,21)$. These assays have nanogram sensitivity, and quantify the total amount of each protein present, whether free or bound. TIMP assays performed on conditioned media containing $10 \%$ FBS were calculated by subtracting $0.02 \mu \mathrm{g} / \mathrm{ml}$ for the small contribution of bovine TIMP that is present in serum minimally cross-reactive with human TIMP antibody.

Gel diffusion was performed in a $1.0 \%$ agar matrix as described by Ouchterlony (22).

Immunoprecipitation of collagenase, TIMP, and gelatinase. Metabolic labeling of newly synthesized bone cell proteins was accomplished by pulsing the osteoblasts with $\left[{ }^{35} \mathrm{~S}\right]$ methionine $(50 \mu \mathrm{Ci} / \mathrm{ml})$ in methionine-free nutrient media for $24 \mathrm{~h}$. Specific polyclonal antisera to human fibroblast collagenase, TIMP, and gelatinase were then utilized for immunoprecipitation as described previously (23).

Functional assay for collagenase. Guinea pig type I collagen was prepared by cold neutral salt extraction as described by Gross (24). Bone cell-conditioned media, collected under serum-free conditions for 24-48 h, were concentrated by either of two methods: $(a)$ dialysis against $2.5 \mathrm{mM}$ Tris, pH 7.5 , containing $0.5 \mathrm{mM} \mathrm{CaCl}_{2}$, and subsequent lyophilization with the samples reconstituted in $1 / 20 \mathrm{vol}$ of water; and $(b)$ ultrafiltration using Centricon 10-kD filters (Amicon Corp., Danvers, MA). Samples were also analyzed without prior concentration. To activate latent collagenase, conditioned media samples were either treated with phenylmercuric chloride (final concentration $1 \mathrm{mM}$ ) or were exposed to trypsin (range $0.01-10 \mu \mathrm{g}$ ) for $10 \mathrm{~min}$ at room temperature followed by the addition of an eightfold molar excess of soybean trypsin inhibitor. Aliquots $(100 \mu \mathrm{l})$ were then incubated with $30 \mu \mathrm{l}$ of collagen $(0.4 \mu \mathrm{g} / \mu \mathrm{l})$ at $25^{\circ} \mathrm{C}$ for $24 \mathrm{~h}$ in buffer containing $0.05 \mathrm{M}$ Tris, $0.01 \mathrm{M} \mathrm{CaCl}_{2}$, and $0.4 \mathrm{M} \mathrm{NaCl}, \mathrm{pH}$ 7.5. The reactions were stopped by the addition of $70 \mu$ l of SDS sample buffer containing EDTA and dithiothreitol, and the samples were boiled immediately. The samples were then subjected to polyacrylamide gel electrophoresis performed according to the method of King and Laemmli (25) with 9.0\% polyacrylamide gels cast in slab molds. After electrophoresis, the protein bands were stained with $1 \%$ Coomassie blue. Samples were also analyzed for the capacity to degrade native, reconstituted collagen fibrils (biosynthetically labelled with $\left[{ }^{14} \mathrm{C}\right]$ glycine) at $37^{\circ} \mathrm{C}$.

Functional assay for TIMP. TIMP was partially purified by subjecting osteoblast conditioned, serum-free media $(200 \mathrm{ml})$ to dialysis against $0.05 \mathrm{M}$ Tris, $\mathrm{pH} 7.5$, and subsequent ion exchange chromatography using a $10 \times 2.5 \mathrm{~cm}$ column of phosphocellulose $(\mathrm{P}-11$, Whatman Inc., Clifton, NJ). TIMP binds avidly to this matrix, whereas serum antiproteases such as $\alpha_{2}$-macroglobulin fall through (26). The bound TIMP was eluted with $0.5 \mathrm{M}\left(\mathrm{NH}_{4}^{\prime}\right)_{2} \mathrm{SO}_{4}$, dialyzed against 0.005 $\mathrm{M}$ Tris, $\mathrm{pH} 7.5$, and lyophilized to achieve a 20 -fold concentration.

The partially purified TIMP preparation was then assayed for the capacity to inhibit pure human fibroblast collagenase (21). Human fibroblast collagenase was purified to homogeneity as described previously (27), activated proteolytically with trypsin, and tryptic activity was terminated by the addition of SBTI. Inhibition of collagenase activity was measured at $37^{\circ} \mathrm{C}$ using native, reconstituted, biosynthetically $\left[{ }^{14} \mathrm{C}\right]$ glycine-labeled guinea pig skin collagen as substrate (28). $50 \mu \mathrm{l}$ of a $0.4 \%$ collagen solution was allowed to form an insoluble substrate gel at $37^{\circ} \mathrm{C}$ overnight. Various concentrations of the bone cell inhibitor were incubated with active collagenase $(25 \mu \mathrm{g} / \mathrm{ml})$ at room temperature for $10 \mathrm{~min}$; the reaction mixtures were then added to the collagen gels and incubated at $37^{\circ} \mathrm{C}$ for 3.5 hours. The reactions were stopped by adding $200 \mu \mathrm{l}$ of buffer followed by centrifugation at $9,000 \mathrm{~g}$ for $10 \mathrm{~min}$. The supernatant fractions were then counted in a 
liquid scintillation spectrometer (Beckman Instruments, Inc., Palo Alto, CA). TIMP concentration in the partially purified bone media was determined by ELISA (21).

$R N A$ isolation and Northern blot analysis. Total cytoplasmic RNA was isolated by guanidinium isothiocyanate extraction according to established procedures (29). The total RNA was fractionated on a $1.2 \%$ agarose gel containing $2.2 \mathrm{M}$ formaldehyde, transferred to nitrocellulose filters, and then hybridized to nick-translated human fibroblast collagenase (30) or human TIMP (31) cDNA probes. Autoradiography was performed using XAR-5 X-Omat AR film (Eastman Kodak Co., Rochester, NY) developed at $-70^{\circ} \mathrm{C}$.

Gelatin zymograms. To determine if human bone cells were capable of secreting metalloproteases with gelatin-degrading activity, aliquots of conditioned media were electrophoresed on 10\% SDS-polyacrylamide substrate gels containing $1 \mathrm{mg} / \mathrm{ml}$ of gelatin (32). Samples were diluted into an equal volume of sample buffer ( $2 \%$ SDS, $0.15 \%$ glycerol, $0.25 \mathrm{M}$ Tris- $\mathrm{HCl}, \mathrm{pH} 6.8,0.1 \%$ bromphenol blue), loaded without boiling, and separated at $4^{\circ} \mathrm{C}$. After electrophoresis, the gels were washed twice for $15 \mathrm{~min}$ in $2.5 \%$ Triton $\mathrm{X}-100$, then once in a fresh solution of $50 \mathrm{mM}$ Tris, $\mathrm{pH} 8.0$, containing $5 \mathrm{mM} \mathrm{CaCl}_{2}$ and 1 $\mathrm{mM} \mathrm{ZnCl}$. Gels were then incubated in the same buffer for $24 \mathrm{~h}$ at $37^{\circ} \mathrm{C}$ to allow for enzymatic digestion of the gelatin substrate. Proteolytic activity was visualized by staining the gels in $0.5 \%$ Coomassie blue R250 containing $30 \%$ isopropyl alcohol and $10 \%$ acetic acid, followed by destaining in water. This technique is extremely sensitive, results in separation of enzyme-TIMP complexes by the SDS, and provides $M_{\mathrm{r}}$ information on the proteases with gelatinolytic activity.

\section{Results}

Characterization of human bone cells: alkaline phosphatase, bone gla protein, and cAMP production. Cultures obtained from bacterial collagenase digestion of human trabecular bone were characterized as osteoblastic by several criteria including high intrinsic alkaline phosphatase activity, secretion of BGP in response to vitamin D, and the capacity for PTH-stimulated cAMP production (2). Cultures were routinely assessed for alkaline phosphatase both histochemically and biochemically. Histochemical staining for alkaline phosphatase was strongly positive in $80.1 \pm 9.2 \%$ of the cells from the various bone preparations. Biochemical assay for alkaline phosphatase resulted in a mean activity $\pm \mathrm{SE}$ of $436.23 \pm 85 \mathrm{nmol} / \mathrm{min}$ per $\mathrm{mg}$ protein. In contrast, human fibroblast cultures exhibited a mean activity of only $0.4 \mathrm{nmol} / \mathrm{min}$ per $\mathrm{mg}$ protein when assayed under the same conditions.

Confluent cultures were assessed for the capacity to secrete BGP in response to $1,25(\mathrm{OH})_{2} \mathrm{D}_{3}(1 \mathrm{nM})$. Radioimmunoassay of supernatants from unstimulated bone cell cultures revealed no detectable BGP, whereas supernatants derived from cultures treated for $48 \mathrm{~h}$ with the vitamin contained $24.6 \pm 3.0$ $\mathrm{ng} / \mathrm{ml}$ BGP. Supernatants from human fibroblast cultures lacked detectable BGP under stimulated or basal conditions.

Finally, the ability of PTH to stimulate intracellular cAMP was assessed. Treatment of bone cell cultures with $1 \times 10^{-7} \mathrm{M}$ PTH for a 10-min period typically resulted in a mean 13-fold increase in cAMP levels $(3.18 \pm 0.41$ vs $39.0 \pm 7.56$ pmol per well). Similar stimulation of fibroblasts was observed $(1.21 \pm 0.06$ vs. $15.83 \pm 2.89$ pmol per well), although to lower absolute levels, consistent with the previously reported presence of PTH receptors on these cells (33).

TIMP, but not collagenase, is a major secreted product of human bone cells. Experiments to determine the collagenolytic profile of human bone cells were conducted by analysis of five newborn and seven adult-derived bone cell cultures. Cells were incubated for 24-72 $\mathrm{h}$ in either $10 \%$ FBS-containing or serum-free medium. Furthermore, the human bone cells were also exposed to PTH, a bone-seeking hormone which is a potent stimulator of bone resorption (34), or PMA, a known stimulator of collagenase production by human fibroblasts $(35,36)$. The conditioned media from the control and treated cultures were then assayed for interstitial collagenase and TIMP by ELISAs utilizing polyclonal antisera specific for each protein. The results (Table I) indicated that bone cells derived from the various bone specimens secreted only negligible or nondetectable amounts of collagenase, but did produce large quantities of TIMP. In some cases, both PTH and PMA appeared to notably enhance TIMP production as compared with control values. Furthermore, the amounts of TIMP secreted by neonatal-derived osteoblasts were often severalfold greater than those observed for the adult cells.

Exposure of serum-containing bone cell cultures (5 specimens) to recombinant IL- $1 \beta$ likewise failed to stimulate the production of collagenase to measurable levels. Total cytoplasmic RNA isolated from these cultures lacked collagenasespecific mRNA as determined by Northern blot analysis (Fig. $1 A$ ). However, the same preparation of total RNA contained abundant TIMP mRNA, as shown in Fig. $1 B$.

Stimulation of one specimen with IL-1 under serum-free conditions did provoke the secretion of a minimal amount of collagenase which was comparable to that seen with PMA $(0.027 \mu \mathrm{g} / \mathrm{ml}$ vs. $0.012 \mu \mathrm{g} / \mathrm{ml}$, respectively). This bone-derived collagenase was a doublet species of $M_{\mathrm{r}} 57,52 \mathrm{kD}$, exhibiting identical electrophoretic migration to human fibroblast collagenase, as shown by subjecting the respective cell types to metabolic labeling and immunoprecipitation of secreted proteins using collagenase-specific antisera (Fig. 2). The amount of collagenase secreted by this confluent bone cell culture was $<5 \%$ of the fibroblast production rate (Fig. 2), as determined by densitometric scanning and ELISA. Two other bone cell cultures stimulated with IL-1 under serum-free conditions failed to produce immunologically detectable amounts of collagenase. Furthermore, when bone cell cultures (two specimens) were treated with $1,25(\mathrm{OH})_{2}$ vitamin $\mathrm{D}_{3}, \mathrm{EGF}$, and combinations of PTH and $1,25(\mathrm{OH})_{2}$ vitamin $\mathrm{D}_{3}$, or EGF and $1,25(\mathrm{OH})_{2}$ vitamin $\mathrm{D}_{3}$ for $48 \mathrm{~h}$, secretion of collagenase into conditioned media remained undetectable, whereas TIMP production was not consistently affected (data not shown).

Since significant amounts of collagenase were not released by bone cells during 24-72 $h$ of incubation with agents that would be expected to stimulate enzyme secretion, conditioned media collected at earlier time points were also examined. Cultures were exposed to PTH and supernatants harvested at $4,8,16,24,48$, and $72 \mathrm{~h}$. Again, no collagenase release was detectable at any time interval (data not shown), indicating that the cells were not secreting and recapturing the enzymea phenomenon previously suggested to occur in rat UMR-106 cells (14). In contrast, TIMP was secreted in a nearly linear fashion by human bone cells in culture for periods up to $72 \mathrm{~h}$ (Fig. 3).

Cell extracts examined at 4, 8, 16, 24, 48, and $72 \mathrm{~h}$ did not contain immunologically detectable collagenase or TIMP. These data most likely reflect the secretory nature of TIMP. The lack of significant intracellular storage is consistent with previously described mechanisms of TIMP biosynthesis in fibroblasts and macrophages $(21,23,37)$.

Human osteoblast TIMP is immunologically identical to human fibroblast TIMP. A double-immunodiffusion assay performed with pure human fibroblast TIMP, partially puri- 
Table I. TIMP and Collagenase Secreted by Human Bone Cells in Culture

\begin{tabular}{|c|c|c|c|c|c|c|}
\hline \multirow[b]{2}{*}{ Tissue } & \multicolumn{2}{|c|}{ Control } & \multicolumn{2}{|c|}{ PTH } & \multicolumn{2}{|c|}{ PMA } \\
\hline & TIMP & Collagenase & TIMP & Collagenase & TIMP & Collagenase \\
\hline & \multicolumn{6}{|c|}{$\mu g / m l$} \\
\hline \multicolumn{7}{|l|}{ Adult } \\
\hline Rib 1 & 0.123 & $0^{*}$ & 0.153 & 0 & 0.227 & 0 \\
\hline Rib 2 & 0.213 & 0 & 0.216 & 0 & - $^{\ddagger}$ & - $^{\ddagger}$ \\
\hline Rib 3 & 0.112 & 0 & 0.105 & 0 & - & - $^{\ddagger}$ \\
\hline Rib 4 & 0.287 & 0 & $-^{\ddagger}$ & $\ldots^{\ddagger}$ & 0.292 & 0 \\
\hline Rib 5 & 0.228 & 0 & 0.216 & 0 & 0.267 & 0 \\
\hline Rib 6 & 1.312 & 0 & 1.531 & 0 & 0.540 & 0.008 \\
\hline Tibia 7 & 0.143 & 0 & 0.183 & 0 & 0.173 & 0 \\
\hline \multicolumn{7}{|l|}{ Neonatal } \\
\hline Femur \& & 0.973 & 0 & 0.733 & 0 & 1.290 & 0 \\
\hline Vertebra 8 & 1.450 & 0 & 3.002 & 0 & 2.586 & 0.008 \\
\hline Femur 9 & 0.548 & 0 & 0.520 & 0 & 0.648 & 0.007 \\
\hline Vertebra 10 & 0.535 & 0 & 0.588 & 0 & 0.520 & 0.006 \\
\hline Vertebra 11 & 0.186 & 0 & 0.218 & 0 & 0.286 & 0 \\
\hline
\end{tabular}

12 different human bone cell preparations, derived from 11 subjects, were examined for the production of TIMP and collagenase as detailed in Methods. Femur 8 and vertebra 8 were from the same neonate source. Culture media containing 10\% FBS were added to confluent cultures of human osteoblasts. Incubations were performed in the presence of PTH $(100 \mathrm{nM})$ or PMA $(10 \mathrm{nM})$, or in the absence of stimulatory factors (control). After $24 \mathrm{~h}$, the conditioned media were harvested and assayed for TIMP and collagenase by ELISA. Data shown represent average values derived from duplicate or triplicate culture wells for each condition. Standard deviations were $<25 \%$. ${ }^{*}$ Values are $<0.005$, the detection limit of the collagenase ELISA. ${ }^{\ddagger}$ Not done.

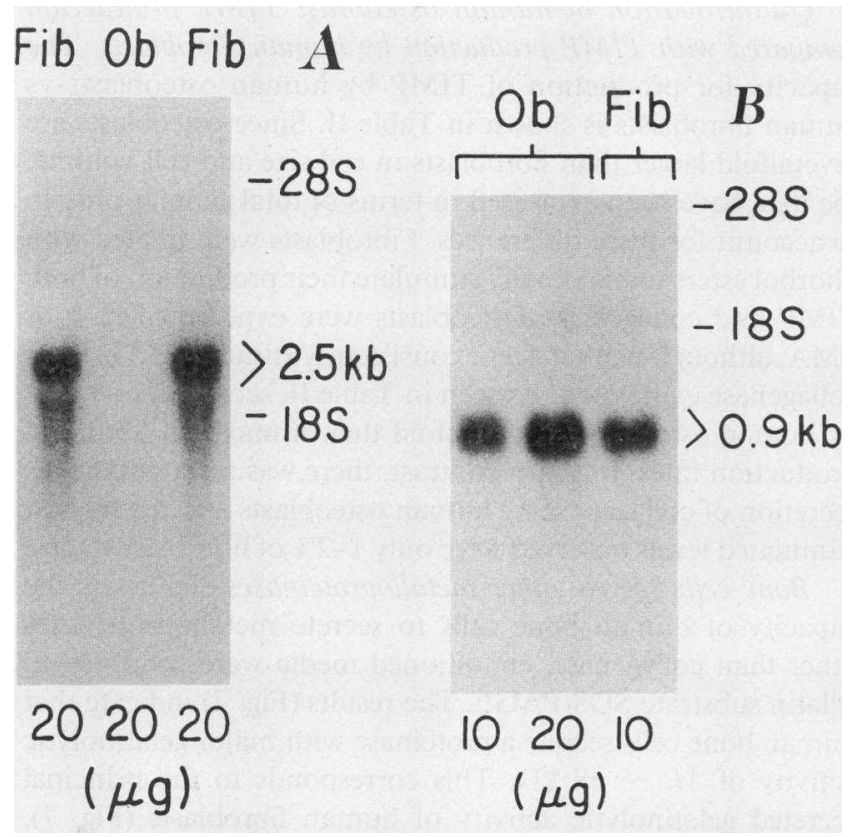

Figure 1. Northern blot analysis of human bone cell and human fibroblast RNA. Human bone cells and human fibroblasts were exposed to IL-1 for $24 \mathrm{~h}$ in serum-containing medium and total cytoplasmic RNA was harvested by guanidinium isothiocyanate extraction as detailed under Methods. 10 or $20 \mu \mathrm{g}$ of osteoblast $(O b)$ or fibroblast (Fib) RNA was applied to the designated slots. (A) Collagenase. The nitrocellulose filter was hybridized to a nick-translated human fibroblast collagenase (30) cDNA probe. (B) TIMP. The nitrocellulose filter was hybridized to a nick-translated human TIMP (31) cDNA probe. The conditioned media from these IL-1-stimulated osteoblasts also lacked immunologically detectable collagenase. Note the absence of collagenase mRNA and the simultaneous abundance of TIMP mRNA in the osteoblast samples. fied bone cell TIMP, and polyclonal antibody to fibroblast TIMP revealed that bone cell cultures contained a protein that was immunologically identical to the fibroblast inhibitor (Fig. 4). Furthermore, when bone cells were metabolically labeled with $\left.{ }^{35} \mathrm{~S}\right]$ methionine and subjected to immunoprecipitation using TIMP-specific antibody, a single protein species of $M_{\mathrm{r}}$ $\sim 28 \mathrm{kD}$ was visualized (Fig. 5), with electrophoretic migration identical to that of fibroblast TIMP. Therefore, human bone cell TIMP appears to be immunologically identical to human fibroblast TIMP and is actively synthesized and secreted by these cells.

Functional assays for collagenase and TIMP. To assess whether bone cells produce a novel interstitial collagenase, immunologically distinct from fibroblast collagenase, a series of functional assays were performed using both native mono-

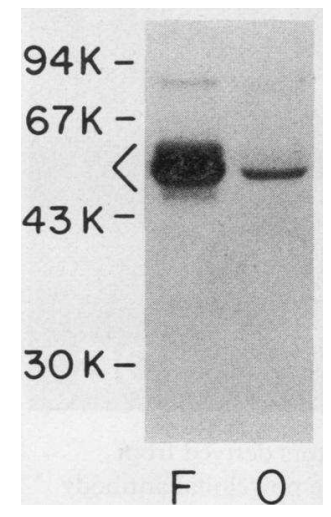

Figure 2. Metabolic labeling and immunoprecipitation of collagenase from human bone cells. Human bone cells stimulated with IL-1 were metabolically labeled with ${ }^{35}$ S]methionine for $24 \mathrm{~h}$ under serum-free conditions. $400 \mu \mathrm{l}$ of labeled osteoblast $(O)$ conditioned media and $200 \mu \mathrm{l}$ of similarly treated human fibroblast $(F)$ media were then immunoprecipitated using collagenase-specific antisera. Note the electrophoretically identical 57,52-kD procollagenase species immunoprecipitated from labeled conditioned media derived from both cell types. The osteoblasts synthesized $<5 \%$ of the collagenase as fibroblasts as determined by densitometric scanning of these autoradiographs. K, kilodalton. 


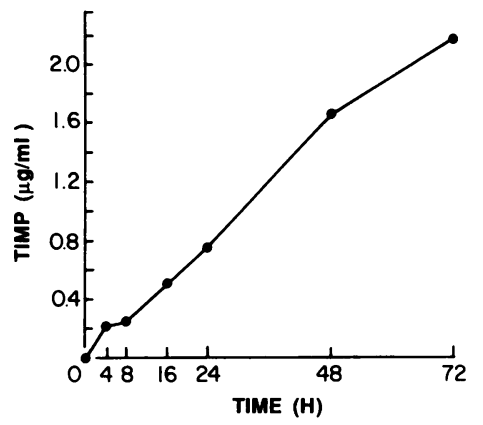

Figure 3. Time course of human bone cell TIMP secretion. Confluent osteoblast cultures were incubated for $4,8,16,24,48$ and $72 \mathrm{~h}$ in serum-containing medium. The conditioned media were then removed and assayed for TIMP by ELISA.

meric type I collagen in solution and ${ }^{14} \mathrm{C}$-labeled collagen fibrils as substrate. Conditioned media samples, concentrated as described in Methods, were derived from bone cell cultures stimulated with PTH, vitamin D, phorbol ester, or combinations thereof. The concentrated samples were assayed with and without exposure to organomercurial compounds or trypsin, agents previously shown to activate the zymogen forms of metalloproteinases secreted by human mesenchymal cells $(27$, 38). The results (data not shown) provided no evidence of type I collagenolytic activity under any condition tested.

Bone cell-derived TIMP was tested for its functional ability to inhibit human fibroblast collagenase. Increasing amounts of partially purified bone-conditioned media were incubated with pure fibroblast collagenase and the resultant collagenolytic activity was measured against ${ }^{14} \mathrm{C}$-labeled collagen fibrils at $37^{\circ} \mathrm{C}$. TIMP present in bone conditioned media was quantified using an ELISA to human fibroblast TIMP. As shown in Fig. 6, the results of this titration indicated that bone-derived TIMP inhibited human collagenase in a stoichiometric manner, with full inhibition observed at a molar ratio of inhibitor:enzyme $\simeq 1: 1$.

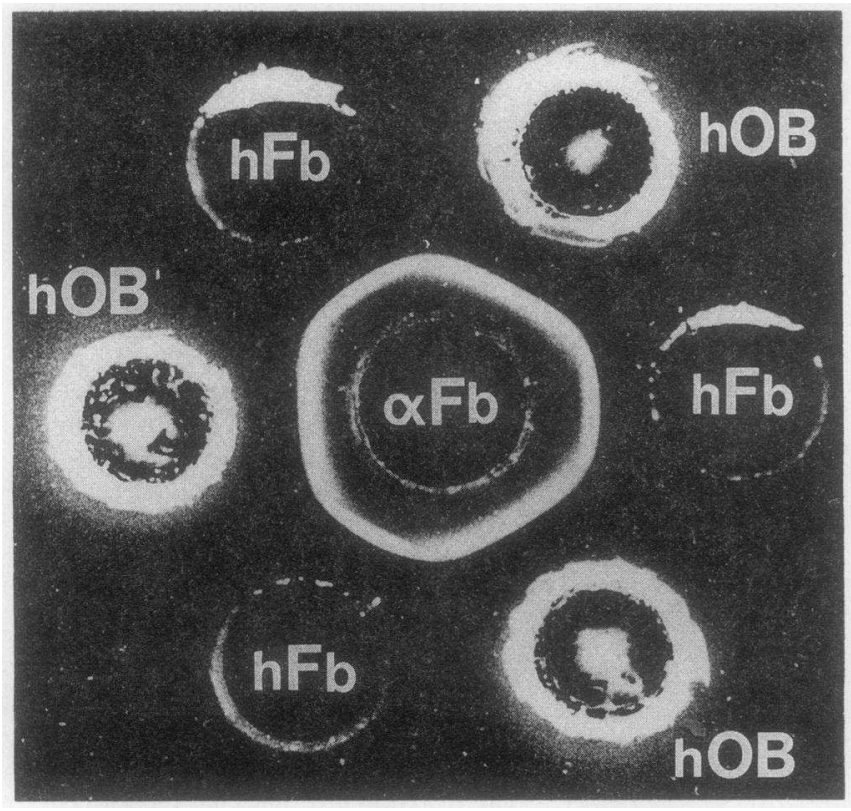

Figure 4. Double immunodiffusion of inhibitors derived from human osteoblasts vs. human fibroblasts. The polyclonal antibody employed $(\alpha F b)$ was prepared against pure human fibroblast TIMP $(21,26) . h O B$, concentrated human osteoblast medium, partially purified as described in Methods; $h F B$, pure human fibroblast TIMP.

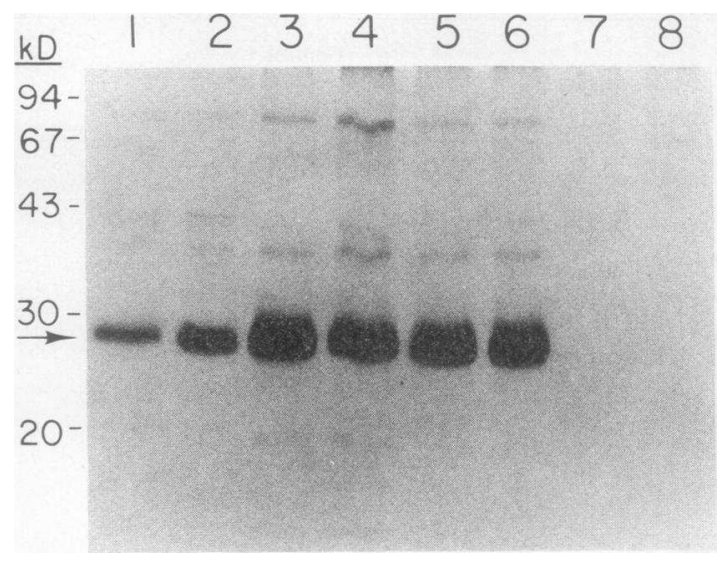

Figure 5. Immunoprecipitation of metabolically labeled TIMP produced by human osteoblasts and human fibroblasts. Human osteoblasts and human fibroblasts were grown to confluency in serumcontaining medium. The cell layers were washed three times and incubated in fresh, serum-free, methionine-free medium containing $\left[{ }^{35}\right.$ S]methionine $(50 \mu \mathrm{Ci} / \mathrm{ml})$ for $24 \mathrm{~h}$. The medium was then harvested and subjected to immunoprecipitation with TIMP-specific antibody as described in Methods. Human fibroblasts: lane 1, control; lane 2, PMA, $1 \times 10^{-8} \mathrm{M}$. Human osteoblasts: lane 3, control; lane 4, rhIL- $1 \beta, 10 \mathrm{U} / \mathrm{ml}$; lane 5, PTH, $1 \times 10^{-7} \mathrm{M}$; lane 6, PMA, 1 $\times 10^{-8} \mathrm{M}$. Lane 7 , fibroblasts, preimmune serum; lane 8 , osteoblasts, preimmune serum.

Quantification of human osteoblast TIMP production compared with TIMP production by human fibroblasts. The capacity for production of TIMP by human osteoblasts vs human fibroblasts is shown in Table II. Since osteoblasts are severalfold larger than fibroblasts in cell size and cell volume, the data have been expressed in terms of total cellular protein to account for these differences. Fibroblasts were treated with phorbol esters to maximally stimulate their production of both TIMP and collagenase. Osteoblasts were exposed to IL-1 or PMA, although neither agent consistently stimulated TIMP or collagenase expression. As seen in Table II, secretion of TIMP by human osteoblasts was twofold that of maximal fibroblast production rates. In sharp contrast, there was no spontaneous secretion of collagenase by human osteoblasts and the highest stimulated levels observed were only $1-2 \%$ of fibroblast values.

Bone cells secrete other metalloproteinases. To assess the capacity of human bone cells to secrete metalloproteinases other than collagenase, conditioned media were subjected to gelatin substrate SDS-PAGE. The results (Fig. 7) indicate that human bone cells secrete a proteinase with major gelatinolytic activity of $M_{\mathrm{r}} \sim 68 \mathrm{kD}$. This corresponds to the principal secreted gelatinolytic activity of human fibroblasts (Fig. 7). Furthermore, the confluent bone cell cultures appeared to secrete quantities of this gelatinolytic activity similar to those from confluent fibroblasts. This proteolytic capacity was completely abolished by EDTA (data not shown). The identity of this bone cell-derived gelatinolytic activity with human fibroblast gelatinase was further established by metabolic labeling of the respective cell types with $\left[{ }^{35} S\right]$ methionine and subsequent immunoprecipitation of the labeled conditioned media using specific antisera directed against human skin fibroblast 68-kD gelatinase (39), which demonstrated the synthesis of identical protein species by both cell types (Fig. 8). These data indicate that although human bone cells do not secrete colla- 


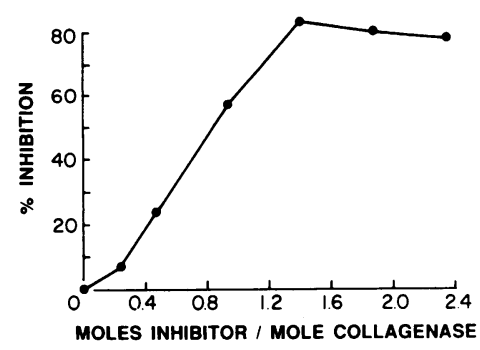

Figure 6. Stoichiometry of human osteoblast TIMP inhibition of human collagenase. Inhibition of pure fibroblast collagenase by concentrated and partially purified human osteoblast medium was quantified as described in Methods. The amount of human osteoblast TIMP was determined by ELISA.

genase, they can secrete other metalloproteinases which may be involved in matrix turnover.

\section{Discussion}

The mechanism(s) by which osteoid is removed from the bone surface, prior to osteoclastic resorption of bone, is still unclear. Several reports have indicated that highly enriched populations of osteoblasts, derived from rodent bone, synthesize and secrete collagenase after exposure to bone-resorbing agents such as PTH and PGE $_{2}(15-18,40-42)$. These findings raised the question of whether human osteoblasts responded to bone-resorbing agents in a like manner to rodent osteoblasts by the secretion of a neutral interstitial collagenase. Our findings indicate that, under the conditions tested, highly enriched populations of human osteoblasts in monolayer culture do not secrete significant quantities of collagenase in response to bone-seeking hormones including agents which have been shown to stimulate either bone formation or bone resorption.

Our search for a bone cell-derived interstitial collagenase was conducted utilizing both immunologic approaches and assays of functional activity. The mainstay of our immunologic methods was an ELISA for human skin fibroblast colla-

Table II. Comparison of the Stimulated Secretion of TIMP and Collagenase from Cultured Human Osteoblasts and Fibroblasts

\begin{tabular}{lcc}
\hline Cell type & TIMP & Collagenase \\
\hline & $\mu g / m g$ protein per $24 h$ & $\mu g / m g$ protein per $24 h$ \\
Osteoblast & $12.91 \pm 2.92$ & $0.19 \pm 0.03^{*}$ \\
Fibroblast $^{\ddagger}$ & $6.02 \pm 2.16$ & $10.21 \pm 2.40$ \\
\hline
\end{tabular}

Osteoblasts were incubated for $24 \mathrm{~h}$ in medium containing 10\% FBS and $10 \mathrm{nM}$ PMA. After the incubation period, the conditioned media were collected and assayed for collagenase and TIMP by ELISA. TIMP values represent the mean \pm SEM of 12 bone cell cultures. Collagenase values, as cited above, represent the mean \pm SEM of only those cultures producing detectable enzyme levels. Human fibroblasts were maximally stimulated by exposure to medium containing 10\% FBS and 10 nM PMA.

* The stimulated values for collagenase are an average of six osteoblast samples derived from four bone cell cultures in which exposure to PMA or IL-1 provided measurable ELISA values. Conditioned media samples from six stimulated bone cell cultures contained undetectable levels of collagenase and are not included in the table. ₹ Fibroblast data are derived from five human skin fibroblast cell lines and have been reported previously (54).

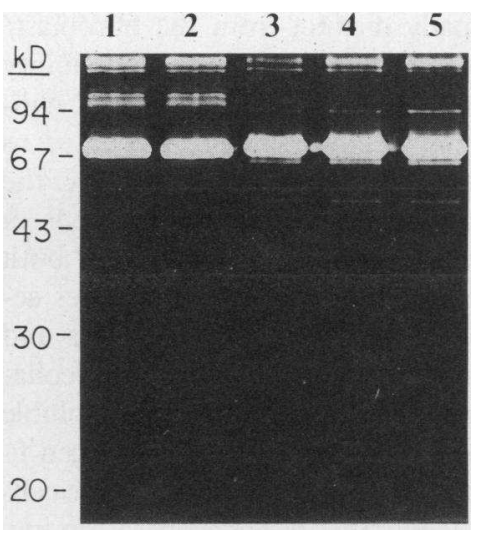

Figure 7. SDS-polyacrylamide substrate gel electrophoresis (gelatin zymogram) of human osteoblast conditioned medium. Confluent cultures of human osteoblasts and fibroblasts were placed in fresh serumfree medium for $24 \mathrm{~h}$ containing: lane 1 , osteoblasts, medium alone; lane 2 , osteoblasts, PTH, $1 \times 10^{-7}$ M; lane 3, fibroblasts, medium alone; lane 4 , fibroblasts, PTH, $1 \times 10^{-7} \mathrm{M}$; lane 5, fibroblasts, PMA, 1 $\times 10^{-8} \mathrm{M}$. The conditioned media were then subjected to polyacrylamide gel electrophoresis using substrate gels impregnated with gelatin as described in Methods. Areas of lysis indicate gelatin-degrading protease activity.

genase that uses specific polyclonal antisera. Such antibody preparations have been employed in the past to demonstrate that human fibroblasts of diverse tissue origin (43), mononuclear phagocytes including peripheral blood monocytes (44) and alveolar macrophages (37), and smooth muscle cells (45) all produce procollagenase species immunologically identical to human skin fibroblast collagenase. This ELISA has a sensitivity of $5 \mathrm{ng} / \mathrm{ml}$, and yet human bone cell cultures released only occasionally detectable levels of collagenase under stimulated conditions (IL-1 and PMA, but not PTH), amounts trivial relative to fibroblast production (Table II). Metabolic labeling and immunoprecipitation experiments confirmed the production of $57,52-\mathrm{kD}$ procollagenase species by these stimulated bone cell cultures, but again in comparatively minor amounts (Fig. 2). Furthermore, pure human fibroblast procollagenase added exogenously to the bone cell cultures under serum-containing or serum-free conditions for $48 \mathrm{~h}$ ( \pm stimulatory agents) was fully detectable by ELISA after the incubation period. Therefore, collagenase was not being secreted and subsequently destroyed in this culture system (data not shown).

Nevertheless, the possibility existed that human osteoblasts synthesized and secreted a collagenase distinct from that secreted by most human mesenchymally derived cells. In this regard, neutrophils have been shown to contain within their specific granules a neutral, interstitial collagenase that is im-

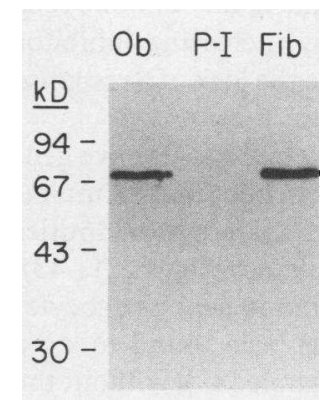

Figure 8. Immunoprecipitation of metabolically labeled $68-\mathrm{kD}$ gelatinase produced by human osteoblasts and human fibroblasts. Human bone cells and human fibroblasts were metabolically labeled with $\left.{ }^{35} \mathrm{~S}\right]$ methionine for $24 \mathrm{~h}$ under basal conditions. $400 \mu \mathrm{l}$ of each conditioned media was then immunoprecipitated using specific antisera to the $68-\mathrm{kD}$ gelatinase produced by human skin fibroblasts (39). Note the production of identical species from both the bone cells $(\mathrm{Ob})$ and fibroblasts (Fib). Immunoprecipitation with preimmune antisera is designated as "P-I." 
munologically and functionally distinct from the fibroblast/ macrophage enzyme $(46,47)$. To investigate the possibility that human bone cells secreted a unique collagenase, the conditioned media from stimulated cultures were subjected to assays of functional collagenolytic activity. Before assay, the media were concentrated and subjected to two methods of activation, trypsin treatment or mercurial treatment, both previously shown to activate latent metalloproteinases secreted in zymogen form $(27,38)$. Both cell supernates and extracts were analyzed in this manner, but functional collagenase activity could not be detected against native, soluble type I collagen or biosynthetically labelled type I collagen fibrils. These results were not affected by exposure of bone cells to phorbol esters, a potent stimulator of collagenase production by fibroblasts $(35,36)$, and furthermore, by exposure to PTH, vitamin D, or combinations of these agents, potent stimulators of bone resorption in vitro (34). Nevertheless, it should be remembered that the presence of TIMP in cell supernates could serve to obscure the detection of metalloproteinasebased collagenolytic activity.

In contrast to the relative lack of human osteoblast secretion of interstitial collagenase, such cells released very substantial quantities of TIMP, both constitutively and after exposure to bone-resorbing agents. The bone cell TIMP was found to be identical to TIMP secreted by human fibroblasts in double immunodiffusion assays (Fig. 4), after metabolic labeling of the cells and immunoprecipitation using TIMPspecific antisera (Fig. 5), and in Northern blot analysis (Fig. 1 $B)$. Furthermore, the bone cell TIMP possessed stoichiometric collagenase inhibitory capacity equivalent to fibroblast TIMP as determined by titration against pure human collagenase (Fig. 6). An intriguing observation in this study was that cells derived from bone of various individuals responded differently to stimulation by PTH (Table I), PMA (Table I), and also IL-1 (not shown) in their secretion of TIMP. Although the extremely slow growth of these bone cells and small numbers available prevented a rigorous examination of TIMP regulation in the present study, it seemed clear that some cultures showed no rise in TIMP secretion while others demonstrated severalfold increases. This individual variability may have important implications in the process of bone resorption and in the natural course of metabolic bone diseases such as osteoporosis, which leads to some individuals losing bone at a slower rate than others within the same age group. Indeed, we have recently reported that elevated levels of IL-1 are associated with high bone formation rates in some individuals with idiopathic osteoporosis (48). These individuals were also found to have accelerated bone loss as judged by computed axial tomography of the vertebral spine. Thus, the ability of bone cells to respond to bone-resorbing agents by increasing inhibitor production may decrease bone loss while the lack of a response could increase bone loss.

Human mesenchymal cells that elaborate TIMP have generally been shown to produce interstitial collagenase simultaneously. Cells releasing both proteins in abundant quantities include human fibroblasts of diverse tissue origin $(21,43)$, smooth muscle cells $(21,45)$, and mononuclear phagocytes $(23,37)$. Thus far, only the platelet has been found to store significant amounts of TIMP in its $\alpha$ granule (49), without the concurrent presence of collagenase. Furthermore, it has been speculated that the simultaneous production of both collagenase and TIMP allows for precise regulatory control of matrix architecture with collagen degradation and synthesis occurring within close proximity as collagen is remodeled in the interstitial space. Our data indicate that the human osteoblast is unusual in its failure to elaborate more than minute amounts of collagenase, while secreting TIMP in quantities exceeding those of human fibroblasts. The present study does not address the precise cellular mechanisms responsible for these observations, but several possible explanations might apply: (a) human osteoblasts, as part of their cellular differentiation, lose the capacity for collagenase synthesis; $(b)$ the correct physiologic stimulator(s) of osteoblast collagenase production was not employed, e.g., a cytokine elaborated by a different cell type; $(c)$ secretion of human osteoblast-derived collagenase requires the coculture of different cell types; $(d)$ collagenase in osteoblasts, unlike collagenase in other mesenchymal cells, is not predominantly secreted but is associated with distinct cellular compartments, e.g., cell membranes; (e) the human bone cell may synthesize a factor that prevents its own production of collagenase; and $(f)$ osteoblasts may be heterogeneous, such that the osteoblast-like cells used in this study represent a subset of cells which do not secrete collagenase, whereas another subset of osteoblasts could accomplish this in vivo. Furthermore, in this study we have examined preparations of bone cells derived from either neonatal or adult tissue. The source of these cells has been bone which develops by the process of endochondral ossification. We cannot exclude the possibility that either fetal bone cells actively participating in bone modeling and/or bone cells derived from tissue which undergoes membranous ossification may be capable of producing collagenase. All of these hypotheses are currently being investigated.

While human osteoblasts fail to elaborate collagenase, they clearly secrete another neutral metalloproteinase in amounts comparable to fibroblasts, a $68-\mathrm{kD}$ enzyme with prominent gelatin-degrading activity. This protease corresponds to the major secreted gelatinase of human fibroblasts (Figs. 7 and 8), and represents a metal-dependent enzyme that has very recently been purified and cloned (39). In addition to denatured collagen, this enzyme has a limited capacity to cleave other extracellular matrix constituents including native types IV and $\mathrm{V}$ collagen, but not type I collagen (39). Its function in bone remodelling is presently unclear but demands further investigation.

The results reported herein raise new questions as to the mechanism of bone resorption in the human as compared to the rodent. Our findings of high levels of TIMP and gelatinase expression by human bone cells contrast sharply with the nonexistent or extremely low levels of secreted collagenase. Although a lack of collagenase activity from human osteoblasts after stimulation with purified IL-1 has been previously reported (50), we have utilized extremely sensitive and specific immunologic probes and have expanded the range of possible stimulators of collagenase secretion to examine human bone cell collagenase production by bone-resorbing agents. Many of the agents tested in this report have been found to stimulate collagenase release from rodent osteoblasts leading to the conclusion that bone cells play a major role in the process of bone resorption by preparing the bone surface for osteoclast attachment. We must conclude, however, that this model may not apply to the process of bone resorption in the human. Indeed, the very agents which would have been expected to stimulate collagenase production in human cells instead stimulated (al- 
beit inconsistently) the synthesis and secretion of TIMP, perhaps suggesting a protective response by the bone cells to stimulators of bone resorption. Thus, we must postulate that cells within the local environment of bone other than osteoblasts, such as macrophages, are possible sources of the collagenolytic activity which has been suggested as necessary for the removal of uncalcified osteoid prior to osteoclastic attachment to the bone, should such a phenomenon be necessary to occur. Since the aforementioned cells do not have PTH receptors, we must further postulate that PTH may induce osteoblasts to secrete a factor(s) which both activates such cells to release collagenolytic activity at the site of bone resorption and also induces resorption of mineralized bone by osteoclasts as suggested by McSheehy and Chambers $(51,52)$ and Thompson et al. (53). In any event, the secretion of TIMP by the bone cells may indeed reflect a protective mechanism to control the amount of osteoid loss. This may account for the existence of only minute foci of osteoclastic action observed in histological sections of bone.

\section{Acknowledgments}

The authors appreciate the excellent laboratory assistance of Ms. Catherine Fliszar, Mr. Charles Dragovich, and Mr. Michael Scott. We also thank Mr. James Havranek for typing the manuscript. The cDNA probe for human fibroblast collagenase and specific antisera to the 68-kD human fibroblast gelatinase were graciously supplied by Dr. Gregory Goldberg, Washington University School of Medicine.

This research was supported by the National Institutes of Health grants AR-32087, AM-35805, and AR-19855. Dr. Welgus is a recipient of Research Career Development Award AM-01525.

\section{References}

1. Nijweide, P. J., E. H. Burger, and J. H. M. Feyen. 1986. Cells of bone: proliferation, differentiation, and hormonal regulation. Physiol. Rev. 66:855-886.

2. Robey, P. G., and J. D. Termine. 1985. Human bone cells in vitro. Calcif. Tissue Int. 37:453-460.

3. Robey, G. H., L. W. F. Shen, M. F. Young, and J. D. Termine. 1988. The biochemistry of bone. In Osteoporosis: Etiology, Diagnosis, and Management. B. L. Riggs and L. J. Melton III, editors. Raven Press, New York. 95-109.

4. Burger, E. H., J. W. M. Van der Meer, J. S. Van de Gevel, J. C. Gribnau, C. W. Thesingh, and R. Van Furth. 1982. In vitro formation of osteoclasts from long-term bone cultures of bone marrow mononuclear phagocytes. J. Exp. Med. 156:1604-1614.

5. Ibbotson, K. J., G. D. Roodman, L. M. McManus, and G. R. Mundy. 1984. Identification and characterization of osteoclast-like cells and their progenitors in cultures of feline marrow mononuclear cells. J. Cell Biol. 99:471-480.

6. Roodman, G. D., K. J. Ibbotson, B. R. MacDonald, T. J. Kuehl, and G. R. Mundy. 1987. 1,25(OH) $)_{3}$ vitamin $\mathrm{D}_{3}$ causes formation of multinucleated cells with several osteoclast characteristics in cultures of primate marrow. Proc. Natl. Acad. Sci. USA. 83:8213-8217.

7. Baron, R., A. Vignery, and M. Horowitz. 1984. Lymphocytes, macrophages and the regulation of bone remodeling. In Bone and Mineral Research, Annual 2. W. A. Peck, editor. Elsevier, Amsterdam. 175-243.

8. Miller, S. S., A. M. Wolf, and C. D. Arnaud. 1976. Bone cells in culture: morphologic transformation by hormones. Science (Wash. DC). 192:1340-1343.

9. Rodan, G. A., and S. B. Rodan. 1984. Expression of the osteoblastic phenotype. In Bone and Mineral Research, Annual 2. W. A. Peck, editor. Elsevier, Amsterdam. 244-285.
10. Rodan, G. A., and T. J. Martin. 1981. Role of osteoblasts in hormonal control of bone resorption-a hypothesis. Calcif. Tissue Int. 33:349-351.

11. Shen, V., L. Rifas, G. Kohler, and W. A. Peck. 1986. Prostaglandins change cell shape and increase intercellular gap junctions in osteoblasts cultured from rat fetal calvaria. J. Bone Miner. Res. 1:243-249.

12. Chambers, T. J., and K. Fuller. 1985. Bone cells predispose bone surfaces to resorption by exposure of mineral to osteoclastic resorption. J. Cell Sci. 76:155-165.

13. Chambers, T. J., J. A. Darby, and K. Fuller. 1985. Mammalian collagenase predisposes bone surfaces to osteoclastic resorption. Cell Tissue Res. 241:671-675.

14. Partridge, N. C., J. J. Jeffrey, L. S. Ehlich, S. L. Teitelbaum, C. Fliszar, H. G. Welgus, and A. J. Kahn. 1987. Hormonal regulation of the production of collagenase and a collagenase inhibitor activity by rat osteogenic sarcoma cells. Endocrinology. 120:1956-1962.

15. Sakamoto, M., and S. Sakamoto. 1984. Immunocytochemical localization of collagenase in isolated mouse bone cells. Biomed. Res. 5:29-38.

16. Sakamoto, S., and M. Sakamoto. 1984. Isolation and characterization of collagenase synthesized by mouse bone cells in culture. Biomed. Res. 5:39-46.

17. Sakamoto, S., and M. Sakamoto. 1984. Osteoblast collagenase: collagenase synthesis by clonally derived mouse osteogenic (MC3T3E1) cells. Biochem. Int. 9:51-58.

18. Sakamoto, S. M., M. Sakamoto, P. Goldhaber, and M. J. Glimcher. 1975. Collagenase and bone resorption: isolation of collagenase from culture medium containing serum after stimulation of bone resorption by addition of parathyroid hormone extract. Biochem. Biophys. Res. Commun. 63:172-178.

19. Heath, J. K., S. J. Atkinson, M. C. Meikle, and J. J. Reynolds. 1984. Mouse osteoblasts synthesize collagenase in response to bone resorbing agents. Biochim. Biophys. Acta. 802:151-154.

20. Cooper, T. W., E. A. Bauer, and A. Z. Eisen. 1982. Enzymelinked immunosorbent assay for human skin collagenase. Collagen Relat. Res. 3:205-216.

21. Welgus, H. G., and G. P. Stricklin. 1983. Human skin fibroblast collagenase inhibitor: comparative studies in human connective tissues, serum, and amniotic fluid. J. Biol. Chem. 258:12259-12264.

22. Ouchterlony, O. 1958. Diffusion-in-gel methods for immunological analysis. Prog. Allergy. 5:1-78.

23. Welgus, H. G., N. L. Connolly, and R. M. Senior. 1986. 12-otetradecanoyl-phorbol-13-acetate-differentiated U937 cells express a macrophage-like profile of neutral proteinases. J. Clin. Invest. 77:1675-1681.

24. Gross, J. 1958. Studies on the formulation of collgen. J. Exp. Med. 108:215-226.

25. King, J., and U. K. Laemli. 1971. Polypeptides of the tail fibres of bacteriophage T4. J. Mol. Biol. 62:465-477.

26. Stricklin, G. P., and H. G. Welgus. 1983. Human skin fibroblast collagenase inhibitor: purification and biochemical characterization. J. Biol. Chem. 258:12252-12258.

27. Stricklin, G. P., E. A. Bauer, J. J. Jeffrey, and A. Z. Eisen. 1977. Human skin collagenase: isolation of precursor and active forms from both fibroblasts and organ cultures. Biochemistry. 16:1607-1615.

28. Nagai, Y., C. M. Lapiere, and J. Gross. 1966. Tadpole collagenase: preparation and purification. Biochemistry. 5:3123-3130.

29. Chirgwin, J. M., A. E. Przybyla, R. J. MacDonald, and W. J. Rutter. 1979. Isolation of biologically active ribonucleic acid from sources enriched in ribonuclease. Biochemistry. 18:5293-5299.

30. Goldberg, G. I., S. M. Wilhelm, A. Kronberger, E. A. Bauer, G. A. Grant, and A. Z. Eisen. 1986. Human fibroblast collagenase: complete primary structure and homology to an oncogene transformation-induced rat protein. J. Biol. Chem. 261:6600-6605.

31. Carmichael, D. F., A. Sommer, D. C. Anderson, C. G. Smith, H. G. Welgus, and G. P. Stricklin. 1986. Primary structure and cDNA 
cloning of human fibroblast collagenase inhibitor. Proc. Natl. Acad. Sci. USA. 83:2407-2411.

32. Birkedal-Hansen, H., and R. E. Taylor. 1982. Detergent activation of latent collagenase and resolution of its component molecules. Biochem. Biophys. Res. Commun. 107:1173-1178.

33. Pun, K. K., C. D. Arnaud, and R. A. Nissenson. 1988. Parathyroid hormone receptors in human dermal fibroblasts: structural and functional characterization. J. Bone Miner. Res. 3:453-460.

34. Raisz, L. G. 1984. Studies on bone formation and resorption in vitro. Hormone Res. 20:22-27.

35. Brinckerhoff, C. E., N. H. Grass, H. Nagase, L. Sheldon, R. C. Jackson, and E. D. Harris, Jr. 1982. Increased level of translatable collagenase messenger ribonucleic acid in rabbit synovial fibroblasts treated with phorbol myristate acetate or crystals of monosodium urate monohydrate. Biochemistry. 21:2674-2679.

36. Clark, S. D., S. M. Wilhelm, G. P. Stricklin, and H. G. Welgus. 1985. Coregulation of collagenase and collagenase inhibitor production by phorbol myristate acetate in human skin fibroblasts. Arch. Biochem. Biophys. 241:26-45.

37. Welgus, H. G., E. J. Campbell, Z. Bar-Shavit, R. M. Senior, and S. L. Teitelbaum. 1985. Human alveolar macrophages produce a fibroblast-like collagenase and collagenase inhibitor. J. Clin. Invest. 76:219-224.

38. Harris, E. D. Jr., H. G. Welgus, and S. M. Krane. 1984. Regulation of the mammalian collagenases. Collagen Relat. Res. 4:493-512.

39. Collier, I., S. Wilhelm, A. Eisen, B. Marmer, G. Grant, J. Seltzer, A. Kroneberger, C. He, E. Bauer, and G. Goldberg. 1988. H-ras oncogene-transformed human bronchial epithelial cells (TBE-1) secrete a single metalloproteinase capable of degrading basement membrane collagen. J. Biol. Chem. 293:6579-6587.

40. Otsuka, K., J. Sodek, and H. Limeback. 1984. Synthesis of collagenase and collagenase inhibitors by osteoblast-like cells in culture. Eur. J. Biochem. 145:123-129.

41. Puzas, E., and J. S. Brand. 1979. Parathyroid hormone stimulation of collagenase secretion by isolated bone cells. Endocrinology. 104:325-327.

42. Thompson, B. M., S. J. Atkinson, J. J. Reynolds, and M. C. Meikle. 1987. Degradation of type-I collagen films by mouse osteoblasts is stimulated by 1,25 dihydroxyvitamin- $D_{3}$ and inhibited by human recombinant TIMP (tissue inhibitor of metalloproteinases). Biochem. Biophys. Res. Commun. 148:596-602.

43. Wilhelm, S. M., A. Z. Eisen, M. Teter, S. D. Clark, A. Kroneberger, and G. Goldberg. 1986. Human fibroblast collagenase: glyco- sylation and tissue-specific levels of enzyme synthesis. Proc. Natl. Acad. Sci. USA. 83:3756-3760.

44. Campbell, E. J., J. D. Cury, C. J. Lazarus, and H. G. Welgus. 1987. Monocyte procollagenase and tissue inhibitor of metalloproteinases: identification, characterization, and regulation of secretion. $J$. Biol. Chem. 262:15862-15868.

45. Roswit, W. T., L. Rifas, M. J. Gast, H. G. Welgus, and J. J. Jeffrey. 1988. Human myometrial smooth muscle collagenase: purification and characterization. Arch. Biochem. Biophys. 262:67-75.

46. Hasty, K. A., M. S. Hibbs, A. H. Kang, and C. L. Mainardi. 1984. Heterogeneity among human collagenases demonstrated by monoclonal antibody that selectively recognizes and inhibits human neutrophil collagenase. J. Exp. Med. 159:1455-1463.

47. Hasty, K. A., M. S. Hibbs, A. H. Kang, and C. L. Mainardi. 1986. Secreted forms of human neutrophil collagenase. J. Biol. Chem. 261:5645-5650.

48. Pacifici, R., L. Rifas, S. Teitelbaum, E. Slatopolsky, R. McCracken, M. Bergfeld, W. Lee, L. V. Avioli, and W. A. Peck. 1987. Spontaneous release of interleukin 1 from human blood monocytes reflects bone formation in idiopathic osteoporosis. Proc. Natl. Acad. Sci. USA. 84:4616-4620.

49. Cooper, T. W., G. P. Stricklin, A. Z. Eisen, and H. G. Welgus. 1985. Platelet-derived collagenase inhibitor: characterization and subcellular localization. Proc. Natl. Acad. Sci. USA. 82:2779-2784.

50. Gowen, M., D. D. Wood, E. J. Ihrie, J. E. Meats, and R. G. G. Russell. 1984. Stimulation by human interleukin 1 of cartilage breakdown and production of collagenase and proteoglycanase by human chondrocytes but not by human osteoblasts in vitro. Biochim. Biophys. Acta. 797:186-193.

51. McSheehy, P. M. J., and T. J. Chambers. 1986. Osteoblastic cells mediate osteoclastic responsiveness to parathyroid hormone. Endocrinology. 118:824-828.

52. McSheehy, P. M. J., and T. J. Chambers. 1986. Osteoblast-like cells in the presence of parathyroid hormone release soluble factor that stimulates osteoclastic bone resorption. Endocrinology. 119:16541659.

53. Thomson, B. M., J. Saklatvala, and T. J. Chambers. 1986. Osteoblasts mediate interleukin 1 stimulation of bone resorption by rat osteoclasts. J. Exp. Med. 164:104-112.

54. Cury, J. D., E. J. Campbell, C. J. Lazarus, R. J. Albin, and H. G. Welgus. 1988. Selective up regulation of human alveolar macrophage collagenase production by lipopolysaccharide and comparison to collagenase production by fibroblasts. J. Immunol. 141:4306-4312. 